\title{
Devil in the details
}

\section{The Innovative Medicines Initiative is an ambitious plan to accelerate the development of new drugs in the European Union. But however worthy, its implementation might prove challenging.}

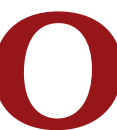
n 20 December, the European Union adopted the Innovative Medicines Initiative (IMI), a venture created by the European Commission and members of the European Federation of Pharmaceutical Industries and Associations (EFPIA; p. 107). The aim of this $€ 2$ billion initiative is to support the faster development of new drugs and to enhance Europe's competitiveness by giving a much-needed boost to its biopharmaceutical sector.

To achieve this goal, the IMI, much like the Critical Path Initiative launched four years ago by the US Food and Drug Administration (FDA), started by asking what bottlenecks make the drug-discovery process so inefficient. And much like the Critical Path Initiative, the IMI found that the drug development industry is not particularly good at predicting the safety of molecules ready to start clinical development and is even worse at predicting their efficacy.

In a 153-page long document, the IMI then outlined its Strategic Research Agenda (SRA) - a series of recommendations to address these and other roadblocks to drug development. Specifically, it made a series of recommendations across four key areas: predictability of safety, predictability of efficacy, resources for knowledge management and resources for education and training.

The money will be used to sponsor projects by public-private partnerships that try to tackle these bottlenecks. The funds contributed by the European Commission ( $€ 1$ billion) will go to academic laboratories and small- and medium-sized enterprises, while the pharmaceutical industry will match this amount by completely funding its own contributions to the partnerships.

It goes without saying that $€ 2$ billion is a welcome lifeline to an industry that has not kept up with that of the United States in terms of both public and venture capital and that is being seriously threatened by emerging economies. For example, whereas the US biotechnology industry invested $€ 12.8$ billion in research and development (R\&D) in 2005, Europe's biotech sector invested only $€ 2.7$ billion. Likewise, China’s R\&D 'intensity' (the ratio of an organization's expenditures on R\&D to its sales) grew 17 times more than the European Union's between 1999 and 2003.
However, once we look at the initiative's details, some problems begin to appear. For starters, the $€ 2$ billion will be spread over a seven-year period (2007-2013) —about $€ 300$ million per year, a very small proportion of, for example, what the European pharmaceutical industry spends on $R \& D$ every year (about $€ 22$ billion in 2005). Moreover, the IMI budget calls for a total of $€ 3.23$ billion for 2007-2013, and the $€ 2$ billion contribution by the European Union and EFPIA falls short of that target by more than $35 \%$. Where will the rest of the money come from?

Setting these financial considerations aside, the heavy involvement of the pharmaceutical industry in the IMI is a double-edged sword. On the one hand, the expertise and resources of EFPIA members will, in principle, enable academic researchers and small- and medium-sized companies to pursue projects that would otherwise be beyond their grasp. On the other, EFPIA members (which include heavyweights such as Merck, SanofiAventis, AstraZeneca and Pfizer) operate in a very competitive market. So, their commitment to the IMI can only exist as long as it doesn't affect the interests of each company.

To solve this conundrum, and to avoid the possibility of being perceived as subsidizing the pharmaceutical industry, the IMI decided to focus on supporting "pre-competitive research"research aimed not at directly developing a drug, but at generating tools that speed up the drug discovery process: biomarker validation, development of better animal models of disease, detection of adverse effects after drug administration, education and training, data integration and analysis tools, and so on.

Thus, more resources will be available to European researchers, but the types of projects they can embark on will be restricted. Although the possibility of applying for IMI funds might be tempting in principle, how many European labs or small companies will be prepared to start a pre-competitive research project that, as the SRA implies in several places, will carry a substantial risk of failure? In all fairness, the IMI's budget also includes funds for research on basic disease mechanisms, but these funds are earmarked for specific conditions (mainly neurological, metabolic and inflammatory diseases) and represent a small fraction of the total investment. For metabolic diseases, for example, the budget allocates less than $€ 100$ million for the entire seven years of the initiative. The lion's 


\section{EDITORIAL}

share of the funds will go to other pre-competitive research projects and to the creation of European centers and "communities of experts" that will work on projects of that kind.

Even if small companies are inclined to solicit IMI funds, the process won't necessarily be straightforward. According to the IMI website, to be eligible, a project needs to be proposed by a partnership of two EPFIA members and two non-EFPIA entities. This requirement is reminiscent of the need to form

European networks as a prerequisite to apply for funds from past Framework Programmes from the European Commission, a painful memory for many researchers who had to enter into "marriages of convenience" in order to qualify for a grant.

In this case, though, the need to form public-private partnerships is an important and welcome aspect of the IMI. Both the FDA's Critical Path and the IMI have made the valid point that these partnerships are necessary to expedite the drug development process. However, their formation raises issues that need to be carefully explored. One of them is the ownership of the intellectual property that results from an IMI-funded research

The doors of perception

\section{What's the difference between real and perceived conflicts of interests?}

project. The IMI has established a series of basic rules for the ownership and the licensing of intellectual property, but ultimately the partners who collaborated on the project are left to agree on who owns what. It may be problematic, to say the least, for a small company or an academic lab to negotiate favorable terms with a large pharmaceutical firm that, in addition to material resources, may also have contributed to the work with its own background intellectual property.

The IMI's creators have put forward a bold vision of how to stimulate Europe's biopharmaceutical sector, and it is now the daunting task of the IMI staff to sort out the details and find the optimal way to implement this vision. Getting it right is crucial for the long-term prospects of this initiative and to make sure that the new investment begins to make a difference for European drug development. But more critically, the importance of getting the IMI to work is to show that public-private partnerships are viable in Europe, making it a more attractive region for venture capital and other forms of public and private investment than it has been so far.
A few weeks ago, a reader of our blog, Spoonful of Medicine, wondered what we meant by 'perceived' conflicts of interest: "This term crops up frequently in the editorials of Nature journals, and I would be extremely grateful if $[\ldots]$ you would like to explain the difference between a perceived and an actual conflict of interest."

This is a frequently asked — and very interesting-question that perhaps only an ethicist could answer in a rigorous way. Editorially, though, let's start by saying that our chief concern relates to conflicts of financial interests (CFIs) and that we have a clear policy about their declaration by authors, referees and editors, which can be found on our website.

In the case of perceived CFIs, we think about them as instances in which no conflict exists, but the potential for financial gain as a result of what we publish is strong enough to give our readers the impression of an actual conflict.

A typical example, from the perspective of our own potential conflicts, is the publication of sponsored content. Producing, say, a supplement to Nature Medicine requires financial resources that may not be part of our budget. If we want to publish this content for the benefit of our readers, we must find the money elsewhere. A sponsor may be interested in the topic of the supplement and agree to underwrite the costs.

To the casual reader, this may look like a CFI-either the sponsor directly paid for the content, or the journal published on this topic to get money from the sponsor. There is, however, no conflict, because our sponsors never have a say on the editorial content of anything we publish. In fact, all of the editorial content for supplements is often already commissioned before we approach potential sponsors.

Our reader, however, is not persuaded: "Even with an absolute division of labor between financial and editorial activity, to be truly unconflicted, an editor would have to be totally unaware of the magazine's readership and advertisers [...]. Conflicts are therefore an inseparable part of editorship, and the important question is whether an editor understands and recognizes conflicts adequately and deals with them satisfactorily."

We agree. In the example above, we would definitely recognize the potential for conflict. Whether we deal with them satisfactorily, though, depends on who you ask, as journals vary a lot in their handling of these issues - there are journals that, as a matter of policy, don't carry sponsored content or even advertising, and there are journals that experience no such inhibition and publish content directly paid for by a sponsor.

In our case, some readers will always construe sponsored content as a CFI, even though we have always striven to be as transparent as possible regarding the role of sponsorship. We are certainly sensitive to these concerns and welcome your views on this matter. 